\title{
Prediction of 3D flow field with non-linear $k-\varepsilon$ model based on unstructured mesh
}

\author{
Hajime NAKAGAWA*, Hao ZHANG**, Taisuke ISHIGAKI*** and Yasunori MUTO**** \\ * Member, Dr. of Eng., Professor, DPRI, Kyoto Univ. (Shimomisu, Yoko-oji, Fushimi-ku, Kyoto 612-8235) \\ ** Member, Doctoral Student, Graduate School of Eng., Kyoto Univ. (Yoshida-Honmachi, Sakyo-ku, Kyoto 606-8501) \\ ***Member, Dr. of Eng., Associate Professor, DPRI, Kyoto Univ. (Shimomisu, Yoko-oji, Fushimi-ku, Kyoto 612-8235) \\ ***** Member, Dr. of Eng., DPRI, Kyoto Univ. (Shimomisu, Yoko-oji, Fushimi-ku, Kyoto 612-8235)
}

\begin{abstract}
An unstructured mesh method is presented for the non-linear $\mathrm{k}-\varepsilon$ model to predict the complex 3D flow filed in engineering practices. The cell-centered FVM (Finite Volume Method) is used for the discretization of the governing equations on a collocated polyhedral mesh. A strict but effective data structure is adopted for the store of the CVs (Control Volumes). It can simplify the application of hybrid polyhedral mesh. Special treatments in the discretization and the boundary conditions have been emphasized. The non-linear model in combination with the wall function approach is employed to a number of turbulent flows. For each test case, the standard $\mathrm{k}-\varepsilon$ model is also implemented for comparison. It is shown that the results of the simulation are in reasunable agreement with those of experiments. Moreover, the non-linear model is always able to give a more accurate result than the standard one.
\end{abstract}

Key Words: non-linear $k-\varepsilon$ model, unstructured mesh, FVM, hybrid polyhedral mesh

\section{Introduction}

Recently, the application of unstructured mesh is undergoing considerable expansion in the community of CFD (Computational Fluid Dynamic). It attributes mostly to some of its inherent advantages. Unstructured mesh offers significant flexibility in treating complicated geometries and provides a convenient means of pursuing mesh adaptation. Both of these are important considerations for the engineering practice to date.

However shifting from structured mesh to unstructured mesh, engineers and researchers have to face a great number of challenges. For example, the boring process of mesh generation, especially the effective generation of arbitrary polyhedra is still not attainable with the current techniques and sometimes becomes the bottleneck of the simulation. The resulted algebraic equation set is usually symbolized by a non-symmetrical, non-positive-definite or even ill-conditioned coefficient matrix. It necessitates the invention of novel solution algorithms. Either of which is worthwhile enough to form a new scientific branch. As the mesh generation and solution algorithms for equation sets involve more mathematics, these subjects are not furthered hereafter.

The last decades have witnessed the rapid rise of LES
(Large Eddy Simulation) and DNS (Direct Numerical Simulation), but these are still under development and a little far from the practical uses for most of the hydraulic engineering applications so far. From the engineering interest and costeffective standpoint, methods derived from the RANS (Reynolds-Averaged Navier-Stokes) equations are preferred. The non-linear $\mathrm{k}-\varepsilon$ model is documented to be a compromise between the ARS (Algebraic Reynolds stress Simulation) model and the standard $\mathrm{k}-\varepsilon$ model. It greatly improves the elaborateness of the standard $\mathrm{k}-\varepsilon$ model with very small increasing of the computational effort. As a result it has achieved a distinctive flourish by the publishing of inexhaustible literatures, e.g. ${ }^{1), 2), 3), 4)}$. Nevertheless, investigation of non-linear $\mathrm{k}-\varepsilon$ models based on unstructured mesh method is still quite few ${ }^{5}$. This has greatly limited the application of non-linear $k-\varepsilon$ models to flows in complex geometries, with moving boundaries or interacting with hydraulic structures.

When unstructured mesh is selected, the connectivity of the mesh has to be explicitly defined. The store of the CVs becomes a problem, especially when the mesh consists of hybrid polyhedra and the number of the CVs is quite large. An object-oriented method is intuitive which includes all the elements of a CV such as faces, edges and vertices, but it might 
result in a vast consumption of computer memories. The discretization of the governing equations is an error-prone process. Comparing with the structured mesh, it is blurred by the mesh skewness and the non-linear terms in the expression of the Reynolds stresses. Disregarding them might sometimes lead to intolerably slow convergence or even undesirable program crash. Furthermore, the boundary conditions pose a more challenging and interesting problem for numerical simulation than their physical appearances. Cautious interpretation and implementation play an important role for a success running. All these problems are tried to solve in this paper.

The proposed methodology is firstly applied to predict the $3 \mathrm{D}$ flow filed in a rectangular channel. After that, it is employed to flows around embayments of different sizes. Different types of unstructured mesh including a hexahedral mesh and a hybrid mesh of hexahedra and prisms have been tested.

\section{Governing Equations}

Turbulence models based on the RANS transform the Navier-Stokes equations in such a way that the transport equations are used for the mean flow quantities only, while the time-averaged statistical turbulent fluctuations are modeled from the information of the mean flow. As engineering practices care more about time-averaged mean values than the transient ones, they have been enjoying a wide spectrum of investigation and application. The representative models of this kind come from the $\mathrm{k}-\varepsilon$ model family based on the eddy viscosity hypothesis.

\subsection{Mean flow field}

The unsteady 3D RANS equations and the continuity equation expressed in a Cartesian coordinate system with the Einstein summation convention are as follows.

Momentum equations:

$$
\frac{\partial u_{i}}{\partial t}+u_{j} \frac{\partial u_{i}}{\partial x_{j}}=f_{i}-\frac{1}{\rho} \frac{\partial p}{\partial x_{i}}+v \frac{\partial^{2} u_{i}}{\partial x_{j} \partial x_{j}}+\frac{1}{\rho} \frac{\partial \tau_{i j}}{\partial x_{j}}
$$

Continuity equation:

$$
\frac{\partial u_{i}}{\partial x_{i}}=0
$$

where $u_{i}=$ time-averaged velocity; $x_{i}=$ Cartesian coordinate component; $\rho=$ density of the fluid; $f_{i}=$ body force; $p=$ time-averaged pressure; $v=$ molecular kinematic viscosity of the fluid; $\tau_{i j}=-\rho \overline{u_{i}^{\prime} u_{j}^{\prime}}$, are the Reynolds stress tensors, and $u_{i}^{\prime}$ is the fluctuating velocity component. As is readily seen, the above equations are not closed because of the unknown Reynolds stress tensors.

\subsection{Turbulence closure}

In order to constitute a relationship between the Reynolds stresses and the mean flow field, different turbulence models have been put forward. Before the description of the detailed models, some definitions are given as below

$$
\begin{aligned}
& S_{i j}=\frac{1}{2}\left(\frac{\partial u_{i}}{\partial x_{j}}+\frac{\partial u_{j}}{\partial x_{i}}\right) \\
& \Omega_{i j}=\frac{1}{2}\left(\frac{\partial u_{i}}{\partial x_{j}}-\frac{\partial u_{j}}{\partial x_{i}}\right) \\
& \delta_{i j}=\left\{\begin{array}{lll}
1 & \text { if } & i=j \\
0 & \text { if } & i \neq j
\end{array}\right. \\
& G=-\overline{u_{i}^{\prime} u_{j}^{\prime}} \frac{\partial u_{i}}{\partial x_{j}}
\end{aligned}
$$

where $S_{i j}=$ the strain-rate tensor; $\Omega_{i j}=$ the rotation-rate tensor; $\delta_{i j}=$ the Kronecker delta; $G=$ the rate-of-production of the turbulence kinetic energy $k$.

In the standard $\mathrm{k}-\varepsilon$ model, the Reynolds stresses are evaluated through the linear constitutive equation

$$
-\overline{u_{i} u_{j}^{\prime}}=\frac{2}{3} k \delta_{i j}-2 v_{t} S_{i j}
$$

where the eddy viscosity $v_{t}$ is evaluated as

$$
v_{t}=C_{\mu} \frac{k^{2}}{\varepsilon}
$$

In the above expression, $C_{\mu}$ is a coefficient, and is usually set to be a constant and equal to 0.09 ; $\varepsilon$ is the dissipation rate of the turbulence kinetic energy, which together with $k$, is computed from the following transport equations.

$$
\begin{aligned}
& \frac{\partial k}{\partial t}+u_{j} \frac{\partial k}{\partial x_{j}}=\frac{\partial}{\partial x_{j}}\left[\left(v+\frac{v_{t}}{\sigma_{k}}\right) \frac{\partial k}{\partial x_{j}}\right]+G-\varepsilon \\
& \frac{\partial \varepsilon}{\partial t}+u_{j} \frac{\partial \varepsilon}{\partial x_{j}}=\frac{\partial}{\partial x_{j}}\left[\left(v+\frac{v_{t}}{\sigma_{\varepsilon}}\right) \frac{\partial \varepsilon}{\partial x_{j}}\right]+\left(C_{1 \varepsilon} G-C_{2 \varepsilon} \varepsilon\right) \frac{\varepsilon}{k}
\end{aligned}
$$

The commonly used model constants are suggested by Rodi $^{\text {()) }}$ and taken the universal values as follows

$$
\sigma_{k}=1.0 \quad \sigma_{\varepsilon}=1.3 \quad C_{1 \varepsilon}=1.44 \quad C_{2 \varepsilon}=1.92
$$

It is well-known that the $\mathrm{k}-\varepsilon$ model has some drawbacks, in particular, the omission of any anisotropic eddy viscosity effects, although it has a widespread use and still vivid nowadays. These defects can be overcome to some extent by introducing a 
non-linear constitutive relation between the turbulence stresses and the mean strain rate satisfying certain tensorial properties.

Introducing quadratic or cubic terms in the standard linear model, a great number of non-linear $\mathrm{k}-\varepsilon$ models have been put forward 1), 2), 4). A general form for a quadratic constitutive equation can be summarized as

$$
\begin{aligned}
& \overline{u_{i}^{\prime} u_{j}^{\prime}}=\frac{2}{3} k \delta_{i j}-2 v_{t} S_{i j} \\
& +a_{1} \frac{k^{3}}{\varepsilon^{2}}\left(\frac{\partial u_{i}}{\partial x_{l}} \frac{\partial u_{l}}{\partial x_{j}}+\frac{\partial u_{j}}{\partial x_{l}} \frac{\partial u_{l}}{\partial x_{i}}-\frac{2}{3} \frac{\partial u_{k}}{\partial x_{m}} \frac{\partial u_{m}}{\partial x_{k}} \delta_{i j}\right) \\
& +a_{2} \frac{k^{3}}{\varepsilon^{2}}\left(\frac{\partial u_{i}}{\partial x_{l}} \frac{\partial u_{j}}{\partial x_{l}}-\frac{1}{3} \frac{\partial u_{m}}{\partial x_{k}} \frac{\partial u_{m}}{\partial x_{k}} \delta_{i j}\right) \\
& +a_{3} \frac{k^{3}}{\varepsilon^{2}}\left(\frac{\partial u_{l}}{\partial x_{i}} \frac{\partial u_{l}}{\partial x_{j}}-\frac{1}{3} \frac{\partial u_{m}}{\partial x_{k}} \frac{\partial u_{m}}{\partial x_{k}} \delta_{i j}\right)
\end{aligned}
$$

where $a_{1}, a_{2}$ and $a_{3}$ are coefficients.

For the verification of the current unstructured mesh method, the model coefficients proposed by Kimura and Hosoda ${ }^{4)}$ is employed in this paper. The model has been tuned by experimental studies and consideration of the constraints of realizability such as non-negativity of the normal Reynolds stresses and Schwarz' inequality between turbulent velocity correlations. With this model, the coefficients are given as below.

$$
\begin{aligned}
& C_{\mu}=\min \left(0.09, \frac{0.3}{1+0.09 M^{2}}\right) \\
& a_{1}=0.0, \quad a_{2}=\frac{0.4 C_{\mu}}{1+0.01 M^{2}}, \quad a_{3}=\frac{-0.13 C_{\mu}}{1+0.01 M^{2}}
\end{aligned}
$$

and

$$
\begin{gathered}
M=\max (S, \Omega) \\
S=\frac{k}{\varepsilon} \sqrt{2 S_{i j} S_{i j}}, \quad \Omega=\frac{k}{\varepsilon} \sqrt{2 \Omega_{i j} \Omega_{i j}}
\end{gathered}
$$

where $S=$ strain parameter and $\Omega=$ rotation parameter.

\section{Numerical Scheme}

Suitable numerical treatments are needed for the solution of the governing equations. Substituting the Reynolds stress expression into the momentum equation, the following equation is obtained.

$$
\begin{aligned}
& \frac{\partial u_{i}}{\partial t}+u_{j} \frac{\partial u_{i}}{\partial x_{j}}=f_{i}-\frac{\partial}{\partial x_{i}}\left(\frac{p}{\rho}+\frac{2}{3} k\right)+ \\
& \left(v+v_{t}\right) \frac{\partial^{2} u_{i}}{\partial x_{j} \partial x_{j}}+v_{t} \frac{\partial}{\partial x_{j}}\left(\frac{\partial u_{j}}{\partial x_{i}}\right)+\frac{\partial N_{i j}}{\partial x_{j}}
\end{aligned}
$$

where $N_{i j}=$ the non-linear terms introduced in the Reynolds stress expression, which is a polynomial of the derivatives of the velocity components.

In the above equation, the terms in the Reynolds stresses have been divided into three parts, the diffusive-like term $v_{t} \partial^{2} u_{i} / \partial x_{j}^{2}$ is treated implicitly as a diffusive term, the term $2 / 3 k \delta_{i j}$ is incorporated into the pressure term and the remainder is transferred to the source terms. If the pressure gradient is also considered as a source term, it is seen that the momentum equations have the same form as that of the transport equations of $k$ and $\varepsilon$.

By satisfying the integral form of the conservation law to a number of continuous polyhedral CVs covering the study domain, the governing differential equations can be integrated using a cell-centered FVM. The general form of the mesh-independent equation over $\mathrm{a} \mathrm{CV}$ is

$$
\frac{\partial}{\partial} \int_{V} \phi d V+\int_{S} \phi \mathrm{r} \mathrm{n} d S=\int_{S} \Gamma \phi \phi \mathrm{n} d S+\int_{V} b d V
$$

where $V=$ the volume of the $\mathrm{CV} ; S=$ the $\mathrm{CV}$ surface with a unit normal vector $\boldsymbol{n}$ directing outwards; $\boldsymbol{\phi}=$ general conserved quantity representing either scalars or vector and tensor field components; $\boldsymbol{u}=$ the fluid velocity vector whose Cartesian components are $u_{i}$ or $(u, v, w) ; \Gamma=$ diffusion coefficient and $b=$ the volumetric source of the quantity $\phi$. The equation has a left side of a transient term and a convective term, which is balanced by a diffusive term and a source term on the right.

\subsection{Numerical mesh}

Although any kind of polyhedral mesh can be used in the simulation theoretically, the generally adopted unstructured mesh in industrial practices is confined to tetrahedra, pyramids, prisms and hexahedra. It might due to the sophistication of mesh generation as well as no certificate of obvious relationship between the mesh complexity and the accuracy improvement. It is noted that among all the above mesh types the hexahedron can be accounted as the general case. (And the quadrilateral is the general case for the surface correspondingly.) Concerning this, the data structure and FVM treatments in this paper are simplified for the hexahedral mesh only, and other polyhedra are considered as hexahedra with some nominal faces. It makes it possible that a hybrid mesh system can be stored with the same data structure irrespective of the type of the polyhedra.

In order to define the connectivity of the CVs, there are a lot of alternatives. As has mentioned before it is possible to define the CV by its six faces, faces by the lists of edges and edges by their corresponding vertices. This method is intuitive and easy to understand, but it is not preferred owing to its great consumption 
of computer memory.

A more restricted but simple data structure is employed here. The CV is simply defined by a list of its eight vertices in a counter-clockwise order. With such definition, the faces enclosing the $\mathrm{CV}$ and the edges forming the faces are also uniquely identified. They can be organized in an ordered way without occupying extra computer memory. Moreover, the neighboring $\mathrm{CV}$ which shares the common face with the current $\mathrm{CV}$ can also be indexed in the same ordered way as that for the faces. For nominal hexahedra, due to the existence of nominal faces, some of the vertices will be repeated during storing. After this kind of treatment, the input of the mesh system includes only a list of nodal coordinates, a list of vertices of the CVs and a list of the neighboring CVs.

\subsection{Spatial Discretization}

For a collocated variable distribution, all the unknowns are defined at the center of the CV. The gradient of the unknown is assumed to be an average over the CV and evaluated with the Gaussian Theorem. For instance, the derivative of $\phi$ with respect to $x$, is evaluated as

$$
\left.\frac{\partial \phi}{\partial x}\right|_{P} \approx \frac{1}{V} \int_{V} \frac{\partial \phi}{\partial x} d V \approx \frac{1}{V} \sum_{f} \phi_{f} S_{f}
$$

where the subscript $P=$ the present $\mathrm{CV}$ and the subscript $f=$ the surface of the CV. With this approximation, the gradient is transformed to a summation of the surface values.

If the transient term is absent for the time being, by employing Eq.13, Eq.12 can be discretized term by term as follows:

$$
\sum_{f} \phi_{f}\left(\mathrm{u}_{f \perp} S_{f}\right)=\left.\sum_{f} \Gamma_{f} \frac{\partial \phi}{\partial n}\right|_{f} S_{f}+b_{P}-S_{P} \phi_{P}
$$

where $u_{f_{\perp}}=$ the fluid velocity normal to the surface; $b_{P}=$ part of the source term containing all the contributions excluding unknown variables and $s_{P} \phi_{P}=$ part of the source term including the unknown variables which can be treated implicitly.

Eq.14, together with Eq.13, is not an explicit expression of the unknowns that defined at the center of the CVs. There are lots of surface values that need to be determined. The commonly used method is an arithmetic interpolation from the values of the $\mathrm{CV}$ on either side, for a quantity $\phi$

$$
\phi_{f}=\alpha_{f} \phi_{P}+\left(1-\alpha_{f}\right) \phi_{A} \text { and } \alpha_{f}=\frac{d_{A f}}{d_{A f}+d_{f P}}
$$

where the subscript $A=$ the adjacent $\mathrm{CV}, d_{f P}$ and $d_{A f}$ are the distances from the surface to the present $\mathrm{CV}$ and to the adjacent $\mathrm{CV}$, respectively. In practices, this method sometimes leads to questionable result, and it is not applied for all the quantities. In this paper, the surface diffusive coefficients, the surface fluxes and the surface values of the conserved quantities are interpolated in some more effective way.

For the evaluation of the diffusive coefficients on the surface, the harmonic mean can reflect more physics and reasonableness. It results in

$$
\Gamma_{f}=\frac{\Gamma_{A} \Gamma_{P}}{\alpha_{f} \Gamma_{P}+\left(1-\alpha_{f}\right) \Gamma_{A}}
$$

A simple arithmetic mean for the fluid velocity on the surface might lead to checkerboard variable distribution, which has caused the collocated mesh out of favor for a long time. This problem can be cured by the interpolation method proposed by Rhie and Chow ${ }^{7}$. The method introduces an additional term related to the pressure gradient when calculating the fluxes on the cell face.

As is known, the unknown quantities of the present $\mathrm{CV}$ can be finally expressed by all of its neighboring CVs after discretization. For instance, the momentum equations for $u$ at present $\mathrm{CV}$ and one of its adjacent $\mathrm{CVs}$ can be written as

$$
\begin{aligned}
& a_{P} u_{P}=\left.\sum_{n b} a_{n b} u_{n b}\right|_{P}-\left.\int_{V} \frac{\partial p}{\partial x} d V\right|_{P}+b_{P} \\
& a_{A} u_{A}=\left.\sum_{n b} a_{n b} u_{n b}\right|_{A}-\left.\int_{V} \frac{\partial p}{\partial x} d V\right|_{A}+b_{A}
\end{aligned}
$$

where $a=$ coefficient for the unknown at the center of the approximated $\mathrm{CV}$ and $n b=$ the neighboring $\mathrm{CV}$.

From the conservation principle of the FVM formulation, the velocity at the common face of the two neighboring CVs must also have a discretized momentum equation of the similar form as that of Eq.17, i.e.

$$
a_{f} u_{f}=\left.\sum_{n b} a_{n b} u_{n b}\right|_{f}-\left.\int_{V} \frac{\partial p}{\partial x} d V\right|_{f}+b_{f}
$$

Approximating the solution $u_{f}$ of Eq.18, the information from Eq.17 can be used. By using some linear interpolation and simplification, the following equation is obtained.

$$
u_{f}=\bar{u}_{f}+\frac{1}{a_{f}}\left(\overline{\left.\int_{V} \frac{\partial p}{\partial x} d V\right|_{f}}-\left.\int_{V} \frac{\partial p}{\partial x} d V\right|_{f}\right)
$$

where

$$
\begin{aligned}
& \bar{u}_{f}=\alpha_{f} u_{P}+\left(1-\alpha_{f}\right) u_{A} \\
& \frac{a_{f}=\alpha_{f} a_{P}}{2\left(1-\alpha_{f}\right) a_{A}} \\
& \left.\int_{V} \frac{\partial p}{\partial x} d V\right|_{f}=\left.\alpha_{f} \int_{V} \frac{\partial p}{\partial x} d V\right|_{P}+\left.\left(1-\alpha_{f}\right) \int_{V} \frac{\partial p}{\partial x} d V\right|_{A} \\
& \left.\int_{V} \frac{\partial p}{\partial x} d V\right|_{f}=S_{f x}\left(p_{A}-p_{P}\right)
\end{aligned}
$$


where $S_{f x}=$ projected area of the surface to the $y z$ plane (perpendicular to the $x$ axis). The extension to other velocity components at the surface is straightforward.

For the conserved quantity $\phi$, evaluation of the surface value is very important for the convective term. Adopting the power law scheme, the finally discretized equation can be assembled as

$$
\begin{aligned}
& \sum_{f}\left\{\left[D_{f} A\left(\left|P_{f}\right|\right)+\max \left(-F_{f}, 0\right)\right]\left(\phi_{P}-\phi_{A}\right)+F_{f} \phi_{P}\right\} \\
& =b_{P}-s_{P} \phi_{P}
\end{aligned}
$$

where the strength of the convection $F_{f}$, diffusion conductance $D_{f}$ and the ratio of them are given as

$$
F_{f}=u_{f \perp} S_{f}, \quad D_{f}=\frac{\Gamma_{f} S_{f}}{d_{A P}}, \quad P_{f}=\frac{F_{f}}{D_{f}}
$$

and

$$
A\left(\left|P_{f}\right|\right)=\max \left\lfloor 0,\left(1-0.1\left|P_{f}\right|\right)^{5}\right\rfloor
$$

\subsection{Mesh skewness}

In the above section, such assumption has been made that the line connecting the neighboring CV centers is almost orthogonal to the cell face and passes through the cell-face center. Under this presumption, the numerical simulation can give almost the same accuracy as that achieved by the mathematical derivation. However it is not always the case. When unstructured mesh is used, the mesh sknewness is almost unavoidable.

Mesh sknewness is generally classified into two kinds: non-conjuctionality and non-orthogonality. The former means that the intersection is not the midway of the surface and the latter stands for a poor perpendicularity. Ferziger and Peric have suggested some ways to maintain the discretization accuracy. ${ }^{8)}$

If a non-conjuctional mesh occurs, the value at the intersection ( $f^{\prime}$ in Fig.1) is firstly evaluated by interpolation, and then a correction term is introduced. The value at the center of the surface ( $f$ in Fig. 1 ) is evaluated from

$$
\phi_{f}=\phi_{f^{\prime}}+(\nabla \phi)_{f^{\prime}} \cdot\left(\mathrm{r}_{f}-\mathrm{r}_{f^{\prime}}\right)
$$

where $f^{\prime}=$ the intersection of the surface and the line connecting

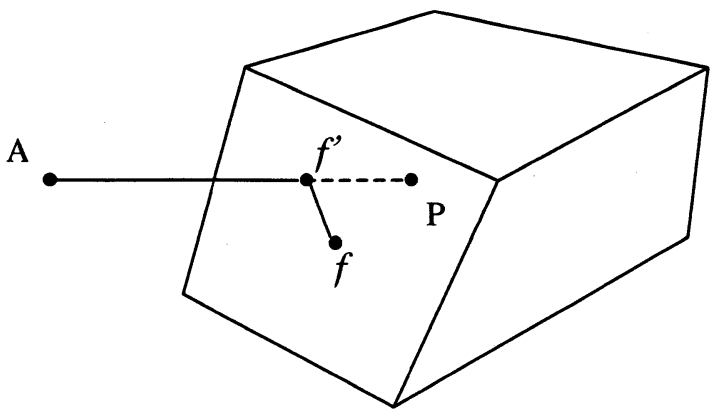

Fig.1 Treatment of non-conjuctionality the two neighboring CVs, the gradient at $f^{\prime}$ is obtained by interpolating the cell-center gradients at either side of the face.

For a non-orthogonal mesh, the normal gradient of a quantity at the surface in the diffusive term has to be corrected. Dividing the diffusion into a normal diffusion and a cross diffusion, the following approximation is suggested.

$$
\left.\frac{\partial \phi}{\partial n}\right|_{f}=\frac{\phi_{A}-\phi_{P}}{d_{A P}}+(\nabla \phi)_{f}^{\exp } \cdot(\mathrm{n}-1)
$$

$$
1=\frac{\mathrm{r}_{A}-\mathrm{r}_{P}}{\left|\mathrm{r}_{A}-\mathrm{r}_{P}\right|}
$$

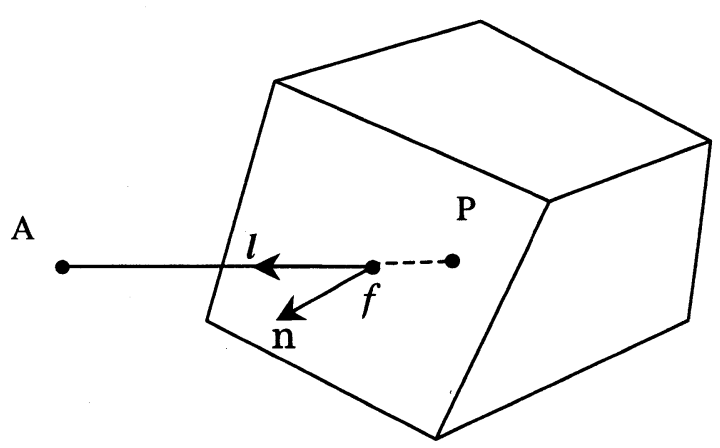

Fig.2 Treatment of non-orthogonality

where $l=$ the unit vector in the direction of the line connecting the center of the current mesh and its neighbor.

The first term on the right hand side is treated implicitly while the second term is a deferred correction which is calculated using interpolated cell center gradients explicitly. (This is the reason why the superscript $\exp$ is used) It is obvious that in the final algebraic equation, the second term becomes a source term. And if non-orthogonality is not very severe, the vectors $\boldsymbol{n}$ and $\boldsymbol{l}$ are co-linear, this source term disappears.

\subsection{Temporal integral}

At the end of the spatial discretization, one can obtain

$$
\frac{\partial}{\partial t} \int_{V} \phi d V=F, \quad F=\sum_{n b} a_{n b} \phi_{n b}+b_{P}-a_{P} \phi_{P}
$$

For a steady calculation, $F=0$, it is the final algebraic equation set. All the coefficients can be acquired from the discretization methods introduced above. In unsteady cases, the temporal integral should be included. The second order implicit Crank-Nicolson Scheme is a suitable solution.

\subsection{Other details}

The resulted equation set is not implemented directly, the under-relaxation method proposed by Patankar ${ }^{8)}$ is adopted 
before the computation. It has been found to be very efficient owing to its increasing of the diagonal dominance of the coefficient matrices.

The algebraic equation systems are solved in an implicit decoupled way. Considering the sparseness and non-symmetry of the coefficient matrices, Krylov subspace iterative methods are preferred. The Bi-CGSTAB $(l)$ (Bi-Conjugate Gradient Stabilized) algorithm suggested by Sleijpen and Fokkema ${ }^{9}$ is employed in this paper. The solution procedure follows the widely used SIMPLE method (Semi-Implicit Method for Pressure-Linked Equations).

\section{Boundary Conditions}

The implementation of boundary conditions requires special attention. As the boundaries do not provide additional equations, introducing of additional unknowns is not desirable ${ }^{8}$. Commonly encountered boundary conditions in engineering practice include the inlet, the outlet, the impermeable wall, the free surface and the symmetrical plane. For the time being, the free surface is considered as a symmetrical plane. This presumption can greatly simplify the solution process and is acceptable for many hydraulic problems.

\subsection{Inlet}

The inlet boundary is considered as a Dirichlet boundary, and all the quantities have to be prescribed. In the discretized equation for the CV near the inlet boundary, as the boundary value is given directly, the contribution from the boundary turns into a source term and no need to be calculated implicitly. In the pressure-correction equation, as the velocity field is given, the velocity correction is zero. And the Neumann boundary of zero gradients is suitable for the pressure.

\subsection{Outlet}

At the outlet boundary, the flow information is usually very little. In order to avoid the propagation of errors, an alternative is to place the outlet boundary as far downstream of the study domain as possible. Then a Neumann boundary with zero gradients can be assumed. In order to ensure the global conservation of mass, the following technique is employed. Firstly, an initial estimation of the velocity at the outlet is acquired by extrapolation from the near boundary CVs. And then the velocity is corrected by making the outlet mass flux the same as the inlet mass flux. A mathematic interpretation is

$$
u_{f i}=u_{f i}^{\text {old }} \frac{\sum_{\text {inlet }} u_{f \perp} \mathrm{S}_{f}}{\sum_{\text {outlet }} u_{f \perp} \mathrm{S}_{f}}
$$

where $u_{f}=$ the velocity component at the outlet boundary ( $i$ ranges from 1 to 3 ) and the superscript old means the value is an initial estimation as mentioned above. As this also provides a way to correct the velocity field at the outlet, the velocity is no need to be corrected again in the SIMPLE procedure.

\subsection{Impermeable wall}

The wall function approach is used near the impermeable wall. Hence the integration through the viscous sub-layer is unnecessary, and meanwhile it makes the consideration and implementation of the wall roughness more easily.

In the wall function approach, it is assumed in this model that the near wall $\mathrm{CV}$ velocity is parallel to the wall and is denoted by $u_{/}$. Although it is not always the case, the treatment can be simplified without significant influence on the result.

With the definition of the dimensionless distance $y^{+}$and dimensionless velocity $u^{+}$as follows

$$
y^{+}=\frac{u_{*} y_{\perp}}{v}, u^{+}=\frac{u_{/ 1}}{u_{*}}
$$

where $u_{*}=$ the friction velocity near the bed and $y_{\perp}=$ the normal distance from the center of the near wall $\mathrm{CV}$ to the wall surface, the universal wall function can be expressed by

$$
u^{+}=\frac{1}{\kappa} \ln \left(E y^{+}\right)
$$

where $\kappa=$ the van Karman constant $(=0.41)$ and $E=$ roughness parameter of the wall. Assuming that the flow is in local equilibrium, i.e. the production and dissipation rate of the turbulence are nearly equal, one can obtain

$$
u_{*}=C_{\mu}^{1 / 4} k_{P}^{1 / 2}
$$

Then the wall shear stress can be written as

$$
\tau_{w}=\rho u_{*}^{2}=\frac{\rho C_{\mu}^{1 / 4} k_{P}^{1 / 2} u_{\| \prime}}{u^{+}}
$$

In the momentum equations, the link with the wall is suppressed by setting it to zero and adding the wall force in Eq.31 as a source term. The normal derivative of $k$ at the wall boundary $\mathrm{CV}$ is set to be zero in the $\mathrm{k}-\varepsilon$ equation, and the production in the wall region is computed from

$$
G_{P}=\frac{\tau_{w}}{\rho} \frac{\partial u_{l \prime}}{\partial n}=\frac{\tau_{w}}{\rho} \frac{u_{\| \prime}}{y_{\perp}}
$$

$\varepsilon$ is not computed in the near wall CV, but is directly set to

$$
\varepsilon_{P}=\frac{u_{*}^{3}}{\kappa y_{\perp}}=\frac{C_{\mu}^{3 / 4} k_{P}^{3 / 2}}{\kappa y_{\perp}}
$$




\section{Model Verification}

In order to verify the applicability of the proposed methodology, the flow in a rectangular channel and the flow around different sizes of embayments are tested with different meshing strategies.

\subsection{Flow in a rectangular channel}

The first verification is the prediction of the 3D flow in a rectangular channel. Detailed measurements were reported by Ishigaki ${ }^{10)}$. An LDV (Laser Doppler Velocimeter) system was used to measure the fully developed turbulent flow velocities in a cross-section. Experimental conditions are given in Table 1.

Table 1 Experimental conditions

\begin{tabular}{|c|c|c|c|c|c|}
\hline $\begin{array}{c}\text { Width } \\
\mathrm{B}(\mathrm{cm})\end{array}$ & $\begin{array}{c}\text { Depth } \\
\mathrm{H}(\mathrm{cm})\end{array}$ & $\begin{array}{c}\text { Discharge } \\
\mathrm{Q}(\mathrm{l} / \mathrm{s})\end{array}$ & $\begin{array}{c}\text { Slope } \\
\mathrm{I}_{\mathrm{e}}\end{array}$ & $\begin{array}{c}\text { Re. } \\
\text { number }\end{array}$ & $\begin{array}{c}\text { Froude } \\
\text { number }\end{array}$ \\
\hline 20.0 & 4.00 & 2.055 & $1 / 1,400$ & 7,700 & 0.48 \\
\hline
\end{tabular}

Considering the geometrical symmetry, only half of the flow field is selected as the computational domain. In order that the influence of the inlet and outlet boundaries can be negligible, a longitudinal distance of $140 \mathrm{~cm}(=35 \mathrm{H})$ has been chosen for the simulation after some trial cases. By using a total number of 10,626 hexahedral mesh, the non-linear $\mathrm{k}-\varepsilon$ model and the standard $\mathrm{k}-\varepsilon$ model are implemented, respectively. A logarithmic velocity profile is assumed at the inlet boundary. The turbulent quantities $k$ and $\varepsilon$ are specified corresponding to a viscosity ratio of 10.0 and taking the turbulence intensity $8 \%$.

The comparison of the longitudinal velocity in the transverse cross-section is given in Fig.3. The linear $\mathrm{k}-\varepsilon$ model does not include any information of the vortices and the secondary flow, which in return affects the prediction of the mean flow pattern. The difference of the longitudinal velocity between the non-linear $\mathrm{k}-\varepsilon$ model and the standard $k-\varepsilon$ model is very obvious. Near the free surface, the non-linear model is able to capture the velocity profile quite well, however there seems to exist great discrepancy between the result of the standard model and the experimental data. Ishigaki also computed the same flow with two ARS models based on structured mesh ${ }^{10)}$. It is interesting to find that the velocity contour predicted by the current non-linear $\mathrm{k}-\varepsilon$ model seems even closer to the LDV measurements than those calculated by the ARS models.

A more reasonable result of the turbulence kinetic energy distribution is again acquired by the non-linear $\mathrm{k}-\varepsilon$ model (see Fig. 4). Nevertheless, both of the computational cases slightly overestimate the turbulence
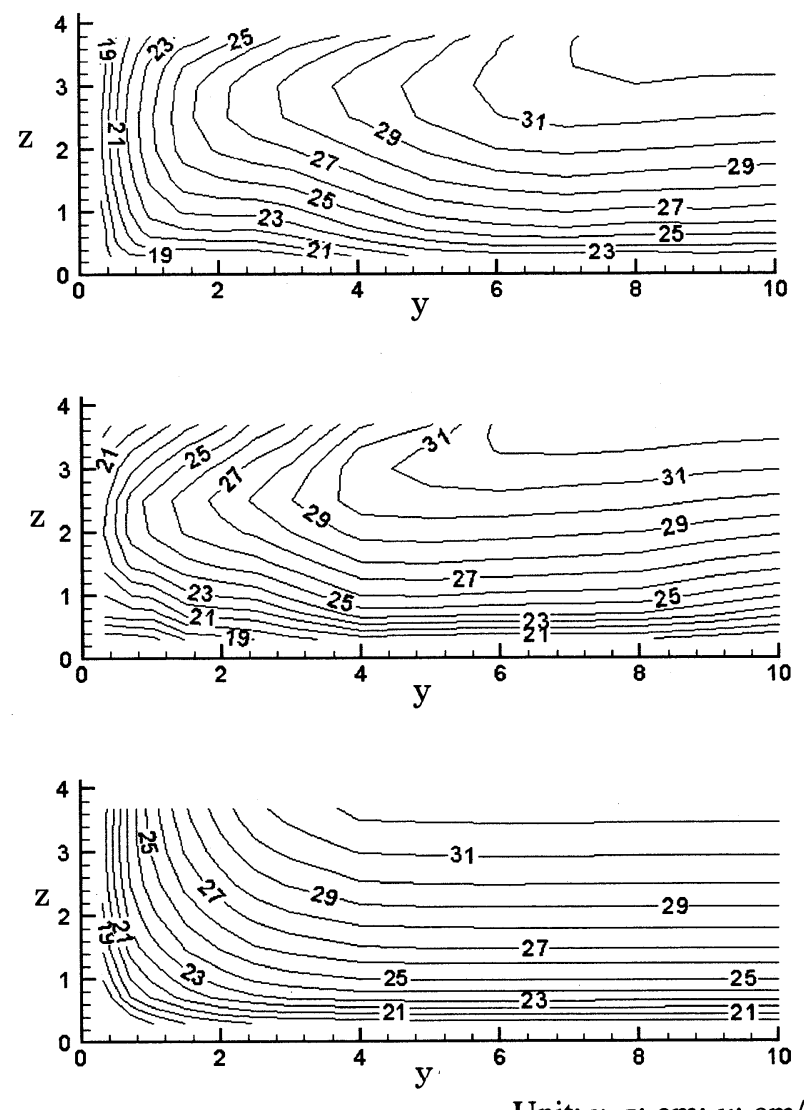

Unit: $y, z: \mathrm{cm} ; u: \mathrm{cm} / \mathrm{s}$

Fig.3 Comparison of the longitudinal velocity $(u)$ :

(Experiment, top; Non-inear, middle; Linear, bottom)
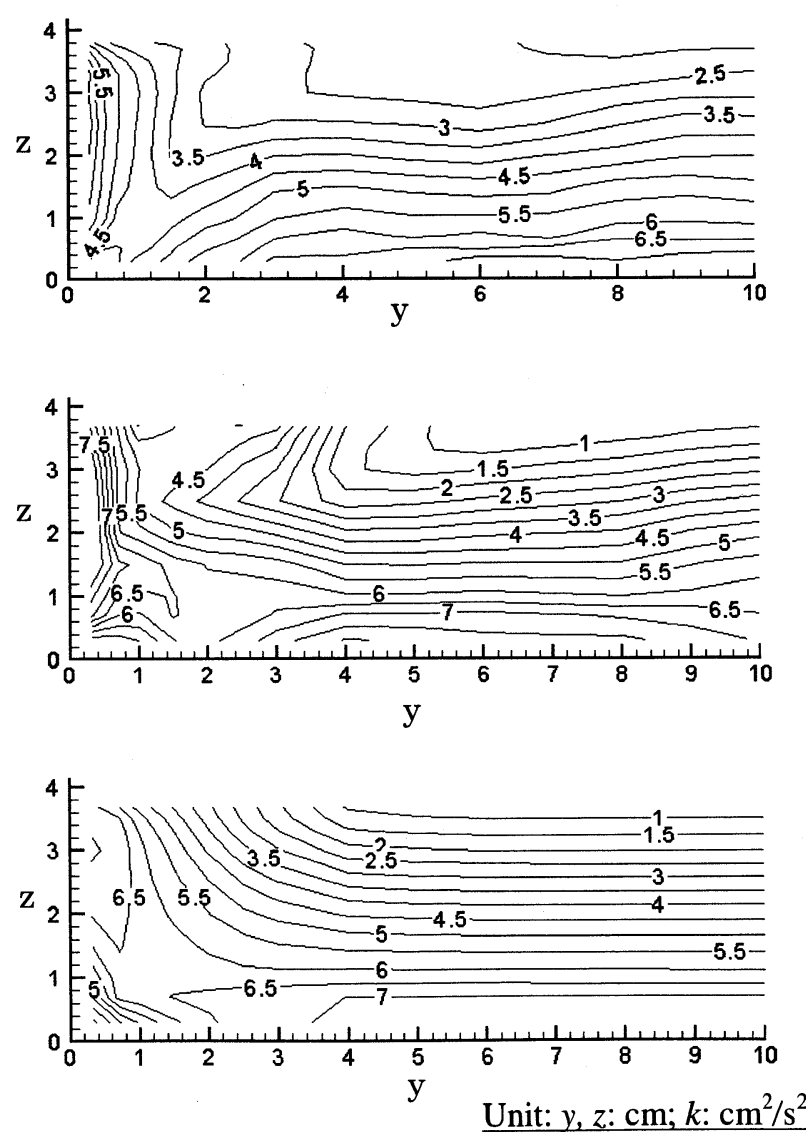

Fig.4 Comparison of the turbulence kinetic energy $(k)$ : (Experiment, top; Non-linear, middle; Linear, bottom) 


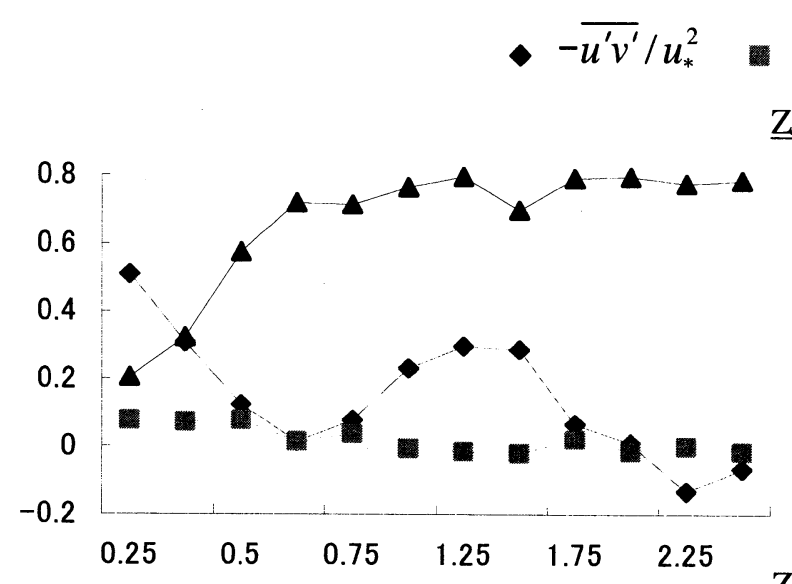

$\underline{\mathrm{Z} / \mathrm{H}=0.25}$
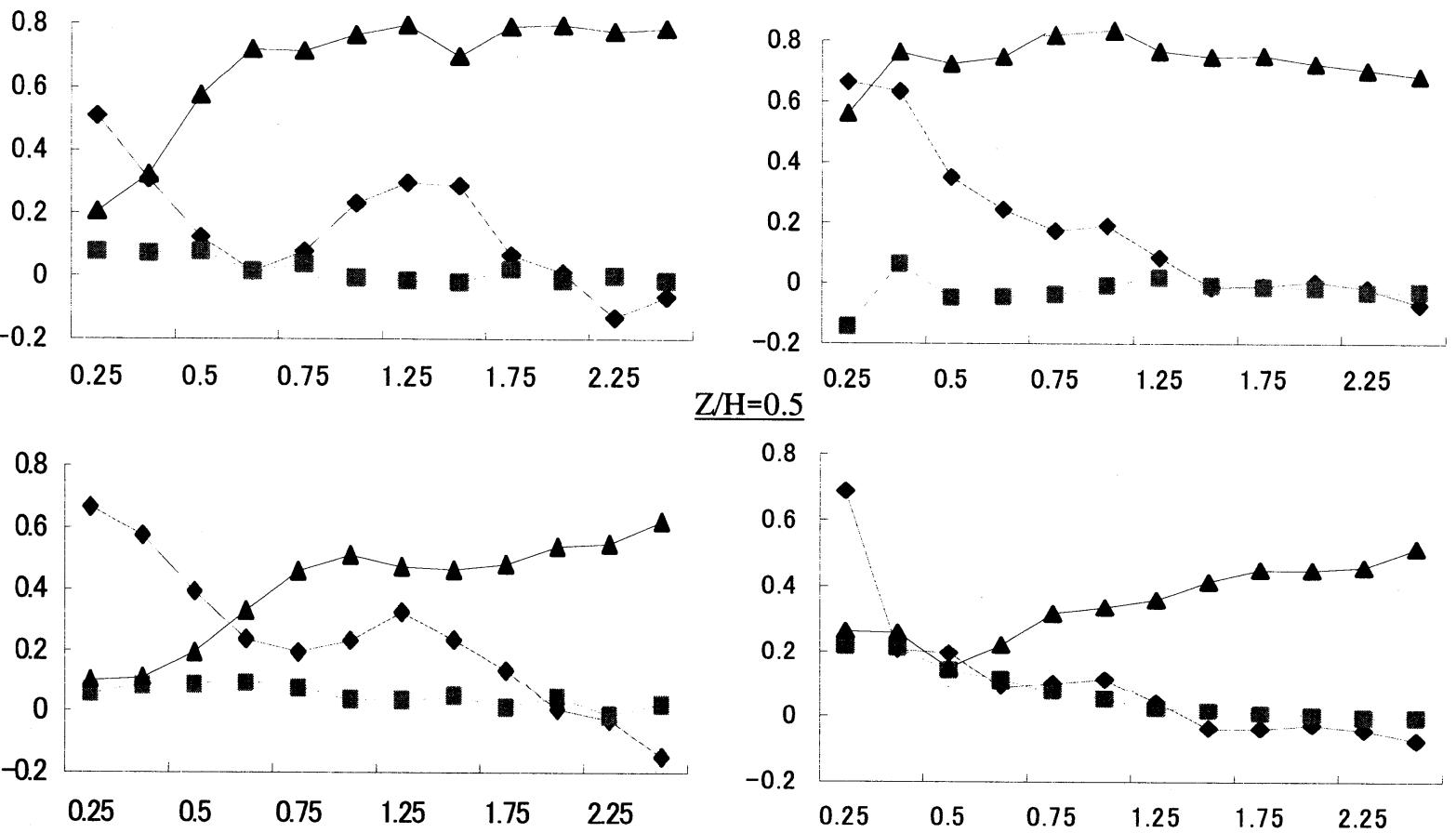

$\underline{\mathrm{Z} / \mathrm{H}=0.5}$

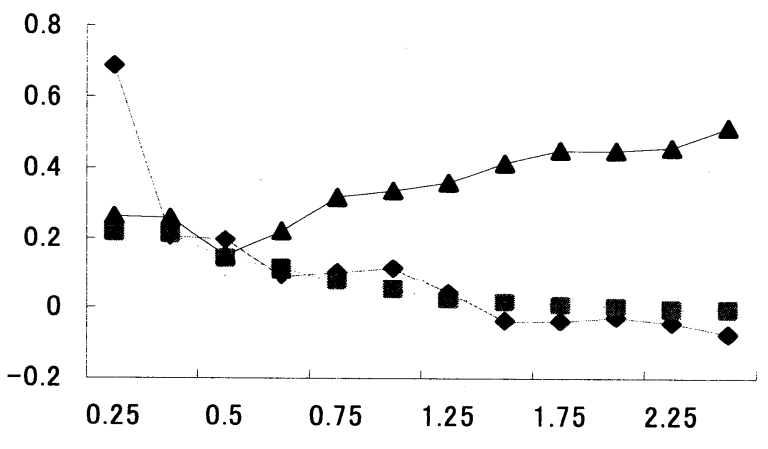

$\underline{\mathrm{Z} / \mathrm{H}=0.75}$
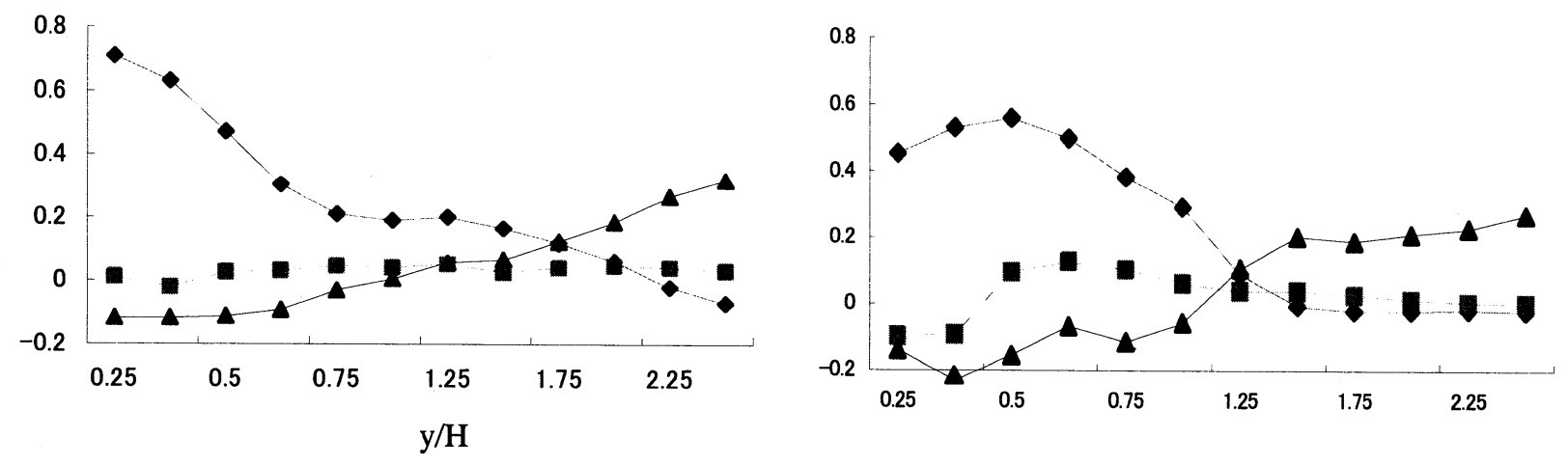

Fig.5 Comparison of the lateral Reynolds stress distribution (Experiment, left; Non-linear model, right)

kinetic energy near the boundary area. A further comparison is carried out for the lateral Reynolds stress distribution at different water depths between the experimental result and the non-linear $\mathrm{k}-\varepsilon$ model. Fig. 5 shows the variation of the Reynolds stresses normalized by $u_{*}^{2}$ (here $u_{*}$ denotes the friction velocity) along the transverse direction. It can be concluded that the Reynolds stresses have been reasonably reproduced in the area where $y / H$ is relatively large. While in the near boundary area (i.e. $\mathrm{y} / \mathrm{H}$ is relatively small), an overestimation can be observed. The exact reason is not very clear yet, but it might be caused by the defects of the wall function approach.

The similarity between the computational results and the experimental data exhibits the applicability of the current unstructured mesh method. The non-linear $\mathrm{k}-\varepsilon$ model is observed to need a little more iteration steps under the same initial and boundary conditions as those of the standard one. But it manifests its advantages over its linear counterpart by more reasonable result.

\subsection{Flow around an embayment}

The 3D flow around the embayment is one of the most important hydraulic phenomena in the river engineering practice. Due to the complex flow structure, the balance of the sediment transport is broken. It results in a great number of problems, for instance, the local scouring, the change of environmental parameters, etc.

In this section, the methodology is applied to this kind of flows. A series of experiments have been carried 
out by Muto et al. ${ }^{11)}$ in a compound flume. The flume consists of a main channel which has a width of $B=16.0 \mathrm{~cm}$ and a flood plain with a width $b=16.0 \mathrm{~cm}$. Part of the flood plain is removed where forms an embayment with a length $L$. The cases of $L=16.0 \mathrm{~cm}$ (i.e. $L=b$ ) and $L=48.0 \mathrm{~cm}$ (i.e. $L=3 b$ ) are validated. The experiment setup is given in Fig.6. The experimental conditions and computational conditions for the verification are shown in Table 2 and Table 3, respectively.

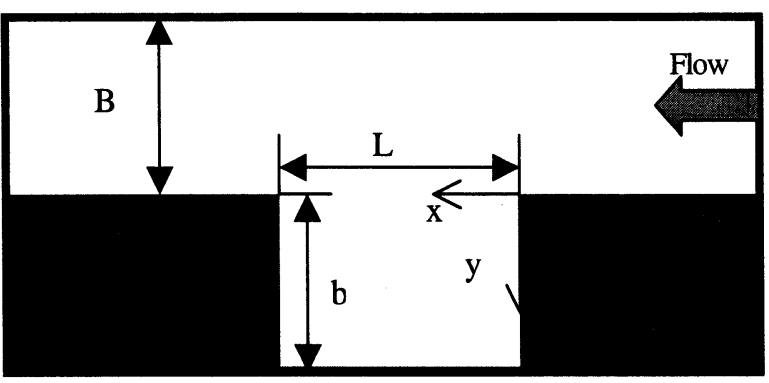

Fig.6 Top view of the experimental setup

The inlet flow is assumed to have a logarithmic velocity profile. At the beginning of the computation, the initial value of the flow is set to be the same as that of the inlet. In order to achieve a compromise between the total mesh number and the influence of the inlet and outlet boundaries, the inlet boundary is set at the distance of about $10 b$ from the embayment, and the outlet, $20 b$.

Table 2 Experimental conditions

\begin{tabular}{|c|c|c|c|c|}
\hline $\begin{array}{c}\text { Discharge } \\
\mathrm{Q}(\mathrm{l} / \mathrm{s})\end{array}$ & $\begin{array}{c}\text { Water } \\
\text { depth } \\
\mathrm{H}(\mathrm{cm})\end{array}$ & $\begin{array}{c}\text { Friction } \\
\text { velocity } \\
u_{*}(\mathrm{~cm} / \mathrm{s})\end{array}$ & $\begin{array}{c}\text { Re. } \\
\text { number }\end{array}$ & $\begin{array}{c}\text { Froude } \\
\text { number }\end{array}$ \\
\hline 2.271 & 3.8 & 1.78 & 9,650 & 0.74 \\
\hline
\end{tabular}

Table 3 Computational conditions

\begin{tabular}{|c|c|c|c|}
\hline Cases & $\begin{array}{c}\text { Aspect ratio } \\
(\mathrm{L} / \mathrm{b})\end{array}$ & Meshing strategy & Number of CVs \\
\hline Case1 & 1 & Hexahedral/Hybrid & $19,683 / 13,086$ \\
\hline Case2 & 3 & Hexahedral & 16,902 \\
\hline
\end{tabular}

\section{(1) Meshing strategy}

Different meshing strategies have been used as described in Table 3. From the standpoint of mesh generation, the hexahedral mesh is easy to be generated for the current study domain. It is adopted for both cases to compare the different flow patterns due to different aspect ratios. In order to investigate the sensitivity of the model to different meshing strategies, a hybrid polyhedral mesh of both hexahedra and prisms is also tested in case1. With this mesh, the areas inside and around the embayment are tessellated by graded prisms, while in areas far from the embayment and near the boundaries, the polyhedral mesh has been used.

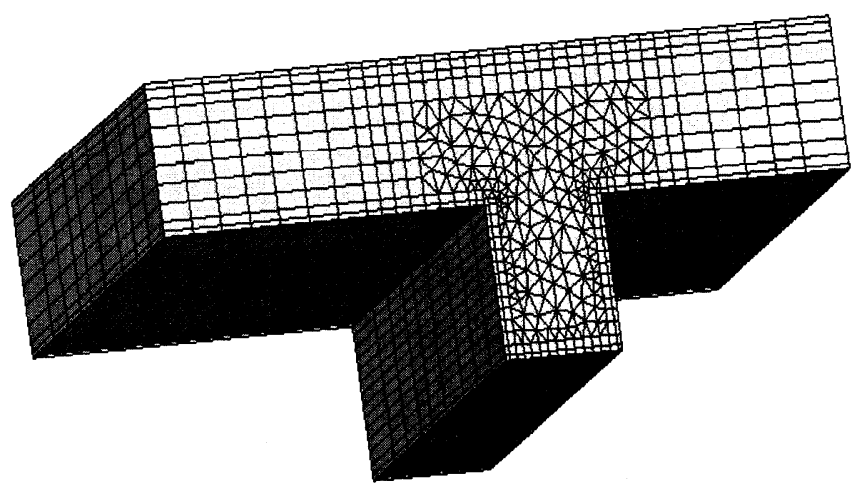

Fig.7 Hybrid polyhedra near the embayment (case1,not to scale)

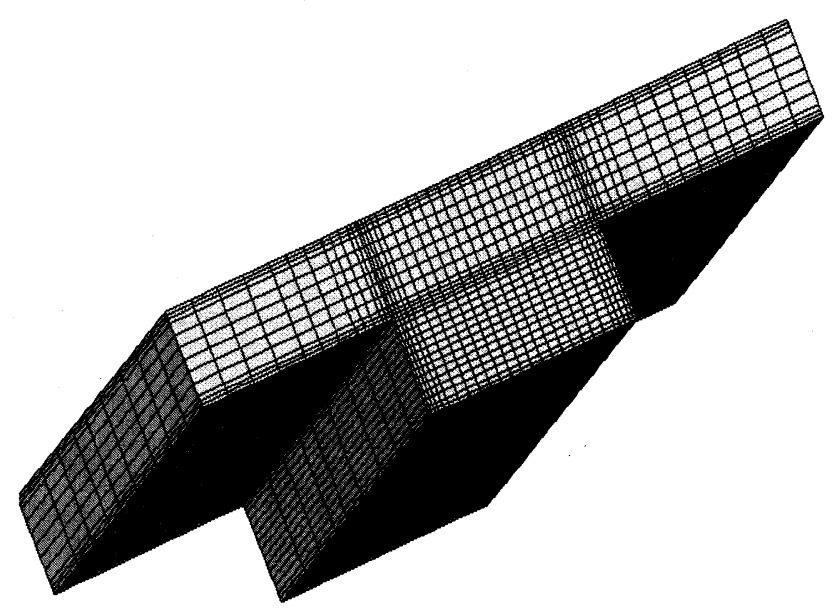

Fig.8 Hexahedra near the embayment (case2,not to scale)

Among all of the meshing methods, the mesh is clustered near the embayment for a better resolution, and away from the embayment the mesh is relatively coarse. Two kinds of mesh structures around and inside the embayment are shown in Fig.7 and Fig.8, respectively.

(2) Horizontal cross-sectional velocity profile $(u, v)$

Comparisons of the computational result and experimental data in the area including the embayment (i.e. the main channel and the embayment area from $x=0$ to $x=L$ ) at $z=1.9 \mathrm{~cm}$ (i.e. half of the water depth) are given as follows.

A large circulating flow is induced in the embayment when aspect ratio $L / b=1$. This circulation occupies the whole embayment, with a very small velocity less than a quarter of the main channel flow. This phenomenon has been well predicted by both the linear and the non-linear models. (See Fig. 9 to Fig. 11) 


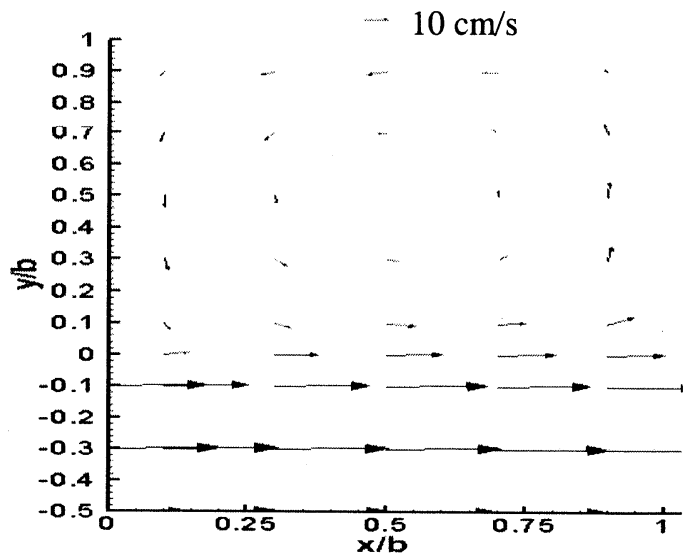

Fig.9 Horizontal cross-sectional velocity profile $(u, v)$ (case1, experiment)

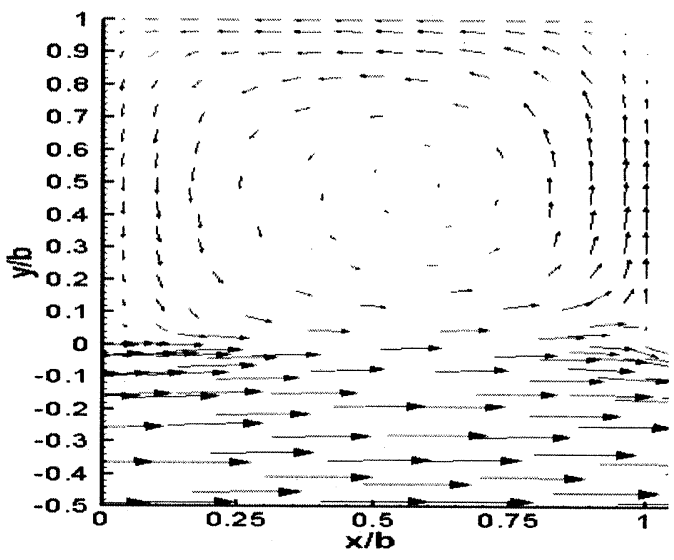

Fig.11 Horizontal cross-sectional velocity profile $(u, v)$
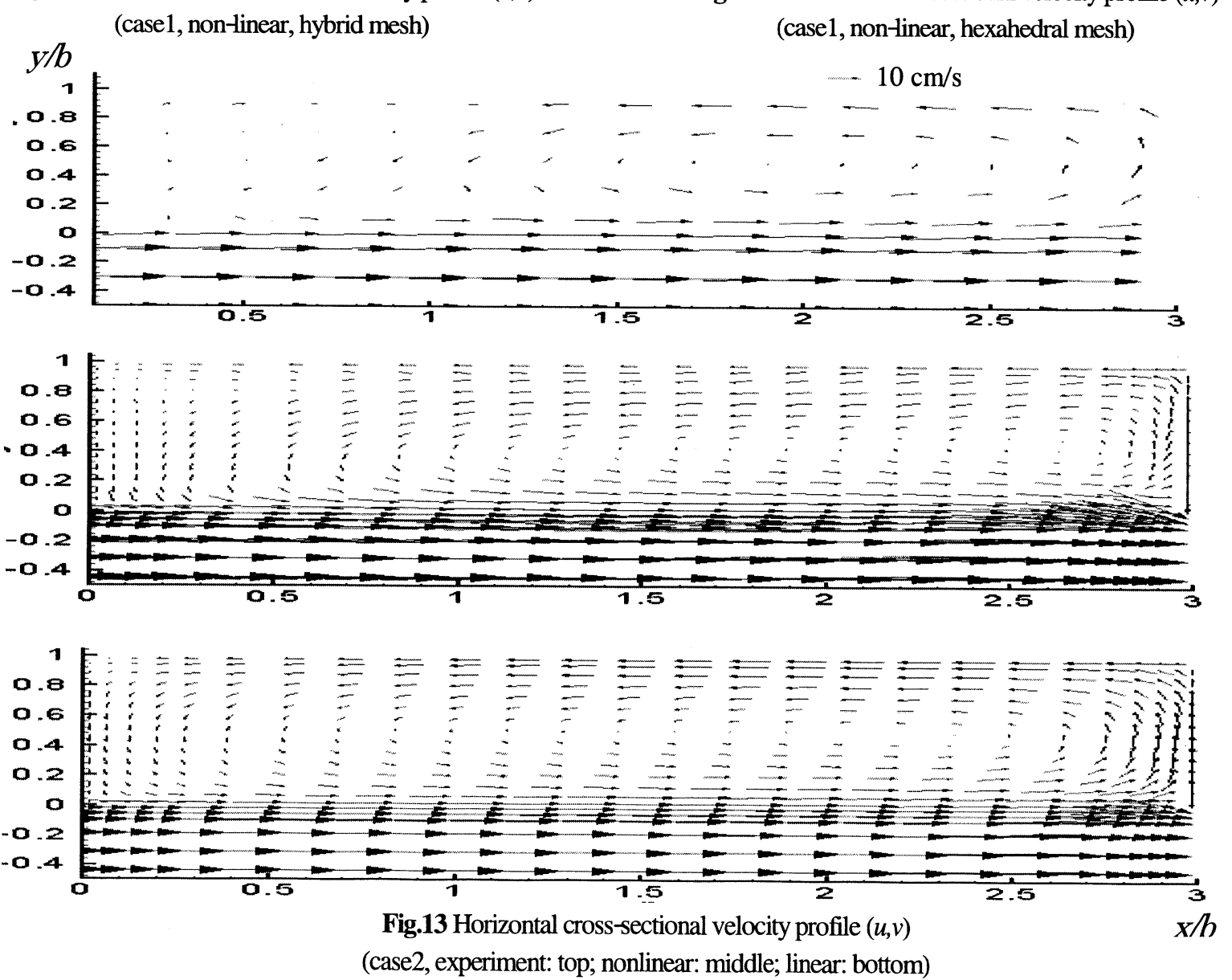
Comparing Fig.11 with Fig.12, one can come to such a conclusion: although different meshing strategies have been performed, very little difference is shown in the final result. It indicates that the treatment for hybrid polyhedral mesh in the current model is effective and applicable.

According to the report of Nakagawa et al. ${ }^{12}$, the number and the shape of the eddies change according to the aspect ratio. This is confirmed by the experimental results of Fig. 9 and Fig.13. In case1, the center of the vortex is almost the same as the geometry center of the embayment. However in case2, due to the increasing of the length, the center of the vortex shifts to the downstream of the embayment very much. In the upstream corner area, there forms a small eddy. But it is very weak comparing with the large one, and this area can be considered as an almost stagnant zone.

The linear model is able to predict the flow pattern in case1, but it fails to capture the eddies in case2. In Fig.13, the linear model gives one large eddy in the embayment although the aspect ratio has increased to 3 . A much larger velocity field has been yielded in the upstream corner area than the experiment. This unreasonableness has been effectively overcome by the non-linear model.

From the above comparison, such conclusions can also be drawn. The non-linear terms in the Reynolds stresses are not influential factors when the aspect ratio $L b$ is small. As the length of the embayment is not long enough for a full mixing of the turbulence, the standard $\mathrm{k}-\varepsilon$ model seems to be applicable. With the increasing of $L b$, the anisotropy of the turbulence becomes more and more important, which has a significant effect on the flow pattern in the embayment. More elaborate models are thus needed for a better resolution. The non-linear $\mathrm{k}-\varepsilon$ model can be a suitable alternative.

\section{(3) Transverse cross-sectional flow pattern $(v, w)$}

Limited to the available experimental data of the vertical velocity component $w$, the comparison of the secondary flow pattern has not been fulfilled directly. As is well-known that the standard $k-\varepsilon$ model could not predict the secondary velocity
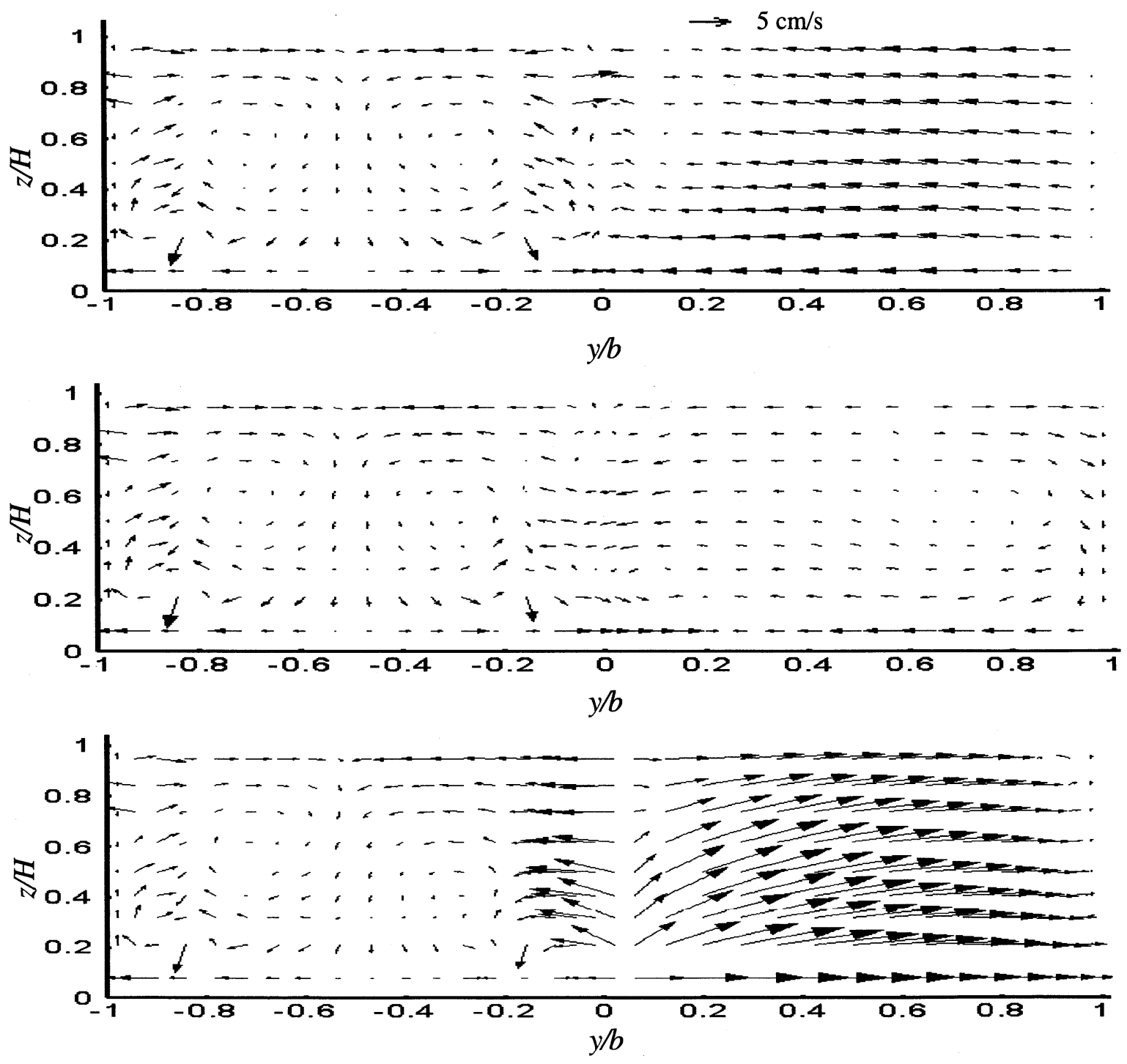

Fig.14 Transverse cross-sectional flow pattern $(v, w)$ (case1, $x=0.3 \mathrm{~cm}$ : top; $x=8.0 \mathrm{~cm}$ : middle; $x=15.7 \mathrm{~cm}$ : bottom) 
vectors. And it is also confirmed by this computation. Taking the anisotropic characteristic of the turbulent flow into account, a more reasonable result has been reproduced by the non-linear $\mathrm{k}-\varepsilon$ model.

Fig.14 shows the computational results of some representative transverse cross-sectional flow in case1. These cross-sections are located at the near-inlet of the embayment $(x=0.3 \mathrm{~cm})$, the center area of the embayment $(x=8.0 \mathrm{~cm})$ and the near-outlet of the embayment $(x=15.7 \mathrm{~cm})$. The propagation of the velocity profile in the cross-sections including the embayment area from the upstream to the downstream can be obviously observed.

In the main channel area, the secondary flow is evident. And the velocity profile almost keeps its shape irrespective of the change of the longitudinal coordinate $x$. In the junction zone, significant momentum exchanges occur. There forms a mixing layer. And the flow velocity field becomes very complex. On both sides of the junction zone, different flow patterns are distinguished. As has mentioned in the previous section, the anisotropy of the turbulent flow in the embayment is not prominent if the aspect ratio $L b$ is not large enough. This can be further observed in Fig.14. Although the variation of the transverse velocity in the embayment area is considerable, the velocity in the vertical direction manifests a very small change from the inlet to the outlet.

\section{Conclusions}

An unstructured mesh method for the non-linear $\mathrm{k}-\varepsilon$ model has been presented and validated against experimental data. With this method, the store of the meshing system is organized by a strict but simple data structure. It is able to take full advantages of the hybrid polyhedral mesh system which is witnessed to become more and more commonplace nowadays. Special treatments distinguished from those of the structured mesh methods have been accentuated including the discretization of the governing equations and the boundary conditions, etc. The algorithm has been confirmed to be both stable and effective. As a preliminary study for a morphological model, some problems involved in the extension from the clear water to the sediment-laden flow have also received particular attention and consideration. As a result, the application to a movable mesh system and inclusion of the bed roughness are straightforward with the current method.

Both the non-linear $\mathrm{k}-\varepsilon$ model and the standard one work well with the proposed methodology. The non-linear $\mathrm{k}-\varepsilon$ model is always able to give a closer result to those of the experiments. It, combining with the unstructured mesh method, can be accounted as a promising solution for the turbulence modeling in hydraulic engineering practices.

\section{Acknowledgement}

This study is financially supported by the Monbukagakusho (Ministry of Education, Culture, Sports, Science and Technology, Japan) under Grant No.14350265.

\section{References}

1) Rubinstein, R. and Barton, J. M., Nonlinear Reynolds stress models and the renormalization group, Physics of Fluids $A 2$ (8), pp.1472-1476, 1990

2) Gatski, T.B. and Speziale, C.G., On explicit algebraic stress models for complex turbulent flows, Journal of Fluid Mechanics, Vol.254,pp.59-78, 1993

3) Kim, S. E., Mathur, S. R., Murthy, J. Y. and Choudhury, D.: A Reynolds-averaged Navier-Stokes solver using an unstructured mesh based finite-volume scheme, Fluent Technical Notes, (TN117) Fluent, Inc., 1997

4) Kimura, I. and Hosoda, T., A non-linear k- $\varepsilon$ model with realizability for prediction of flows around bluff bodies, International Journal for Numerical Methods in Fluids, John Wiley \& Sons, Ltd., Vol.42, pp.818-837, 2003

5) Basara, B., Employment of the second-moment turbulence closure on arbitrary unstructured grids, International Journal for Numerical Methods in Fluids, John Wiley \& Sons, Ltd., Vol.44, pp.377-407, 2004

6) Rodi, W., Turbulence Models and Their Application in Hydraulics - a State of the Art Review, University of Karlsruhe, Karlsruhe, Germany, 1980

7) Rhie, C.M. and Chow, W.L., Numerical study of the turbulent flow past an airfoil with trailing edge separation, Journal of ALAA, Vol.21, pp.1525-1532, 1983

8) Ferziger, J.H. and Peric, M., Computational Methods for Fluid Dynamics, $3^{\text {rd }}$ rev. ed., Springer-Verlag, 2002

9) Sleijpen, G. L. G. and Fokkema, D. R., BiCGSTAB (l) for linear equations involving unsymmetrical matrices with complex spectrum, Electronic Transactions on Numerical Analysis, Kent State University, Vol. 1, pp. 11-32, 1993

10) Ishigaki, T., Study on 3D Flow Structures in Compound Open Channels, Ph. D. Thesis, Kyoto University, 1993 (in Japanese)

11) Muto, Y., Imamoto, $H$. and Ishigaki, T., Velocity measurements in a straight open channel with a rectangular embayment, Proc. $12^{\text {th }}, A P D H A H R$, Bangkok, Thailand, 2000

12) Nakagawa, K., Kawahara, Y. and Tamai, N., Experimental study on hydraulic characteristics of flows in embayments, Annual Journal of Hydraulic Engineering, JSCE, Vol.39, pp. 595-600, 1995 (in Japanese)

(Received: April 16, 2004) 